\title{
Superconducting thin rings with finite penetration depth
}

\author{
Ernst Helmut Brandt \\ Max-Planck-Institut für Metallforschung, D-70506 Stuttgart, Germany \\ John R. Clem \\ Ames Laboratory - DOE and Department of Physics and Astronomy, Iowa State University, Ames Iowa 50011, USA
}

(Dated: November 19, 2018)

\begin{abstract}
Recently Babaei Brojeny and Clem [Phys. Rev. B 68, 174514 (2003)] considered superconducting thin-film rings in perpendicular magnetic fields in the ideal Meissner state with negligibly small magnetic penetration depth and presented useful analytical limiting expressions and numerical results for the magnetic-field and sheet-current profiles, trapped magnetic flux, self-inductance, magnetic moment, and focusing of magnetic flux into the hole when no net current flows in the ring. The present paper generalizes all these results to rings with arbitrary values of the two-dimensional effective penetration depth $\Lambda=\lambda^{2} / d$ ( $\lambda$ is the London depth and $d<\lambda / 2$ the film thickness) using a straightforward matrix inversion method. We also present results for the energy of a superconducting ring as a function of the applied magnetic induction $B_{a}$ and the quantum number $N$ defining the size of the fluxoid $N \phi_{0}$ trapped in the hole.
\end{abstract}

PACS numbers: 74.78.-w, 74.25.Ha,74.25.Op

\section{INTRODUCTION}

In a recent paper Babei Brojeny and Clem ${ }^{\underline{1}}$ calculated the magnetic properties of superconducting thinfilm rings and disks in the ideally screening Meissner state when a perpendicular magnetic field is applied. They showed that the Biot-Savart law for the sheet-current density $\mathbf{J}(r)=\int_{-d / 2}^{d / 2} \mathbf{j}(r, z) d z(\mathbf{j}$ is the current density) in a circular disk of thickness $d$ and radius $b$ with a hole of radius $a(0 \leq a<b)$ can be solved by a physical ansatz containing a factor $\left[\left(r^{2}-a^{2}\right)\left(b^{2}-r^{2}\right)\right]^{-1 / 2}$ describing the divergence of $J(r)$ at the inner and outer radii. A plethora of useful numerical results and analytical limiting expressions is given in Ref. 1 for several situations: (a) magnetic flux trapped in the hole when no magnetic field is applied; (b) zero magnetic flux in the hole when the ring is subjected to an applied field; and (c) focusing of the magnetic flux into the hole when a magnetic field is applied but no net current flows around the ring.

Throughout the paper ${ }^{1}$ it was assumed that either the London magnetic penetration depth obeys $\lambda<d / 2$ or, if $\lambda>d / 2$, the two-dimensional (2D) penetration depth $\Lambda=\lambda^{2} / d$ (or screening length $2 \lambda^{2} / d$ ) is negligibly small. The same assumption was made in previous work on disks ${ }^{2.3 .4}$ and rings .5 .6 However, while thin superconducting rings with $\lambda=0$ ideally screen magnetic flux from penetrating the hole, a finite $\lambda$ or $\Lambda=\lambda^{2} / d>\lambda$ will allow magnetic flux to penetrate into the film as well as the hole. This effect is much stronger than would be suggested by the exponential factor $\exp [-(b-a) / \lambda]$ that applies to long tubes in an axial field.

The effect of finite $\Lambda$ is particularly important for the interpretation of experiments that try to confirm the cosmological Kibble-Zurek mechanism ${ }^{7.8}$ of spontaneous formation of vortices during rapid cooling of a superfluid; some of these experiments use superconducting rings, 9 while others use disks $\frac{10}{2}$ Finite $\lambda$ also modifies flux focusing, an important feature of SQUIDs (superconducting quantum interference devices), which usually have roughly the shape of a washer with a small central hole but with a slit that allows magnetic flux to enter the hole and causes zero net circulating current at the SQUID's critical current 11.12 .13 .14 The concentration of magnetic flux into this hole increases the effective area of the SQUID.

This paper is organized as follows. In Sec. II we describe our calculation method, which applies to arbitrary $\Lambda$. In Sec. III we compute the self-inductance of a thin flat ring. In Sec. IV we calculate the response of a ring in an applied magnetic field. In Sec. V we study the flux-focusing problem and calculate the effective area. In Sec. VI we calculate the energy of a ring as a function of the applied field and the quantum number $N$ describing the size of the fluxoid $N \phi_{0}$ trapped in the ring. In Sec. VII we give some analytical results for the limit of large $\Lambda \gg b$, which applies to mesoscopic rings. We present a brief summary in Sec. VIII.

\section{CALCULATION METHOD}

We assume for simplicity that current in a circular coil far from the ring produces a vector potential $\mathbf{A}_{a}(\mathbf{r})=$ $A_{a}(\mathbf{r}) \hat{\varphi}$, which describes the magnetic induction $\mathbf{B}_{a}=$ $\nabla \times \mathbf{A}_{a} ; \hat{\varphi}$ is the azimuthal unit vector. Near the film in the plane $z=0$, we assume $A_{a}(r)=r B_{a} / 2$, such that the magnetic induction applied to the thin-film ring is $\mathbf{B}_{a}=B_{a} \hat{z}$. In response to either the applied field or a fluxoid trapped in the hole, currents are induced in the ring. The net magnetic induction is $\mathbf{B}(\mathbf{r})=\mathbf{B}_{a}(\mathbf{r})+$ $\mathbf{B}_{J}(\mathbf{r})$, where $\mathbf{B}_{J}(\mathbf{r})=\nabla \times \mathbf{A}_{J}(\mathbf{r})$ and its vector potential $\mathbf{A}_{J}(\mathbf{r})$ are generated by the currents in the film. Because of the circular symmetry, the sheet current in the film has only a $\varphi$-component, $\mathbf{J}(x, y)=J(r) \hat{\varphi}$. Similarly, the 
vector potential $\mathbf{A}(x, y, z)$, defining the total magnetic induction $\mathbf{B}=\nabla \times \mathbf{A}$, has only a $\varphi$-component. In the film plane $z=0$, we have $\mathbf{A}(x, y, 0)=A(r) \hat{\varphi}$ and $A(r)=$ $A_{a}(r)+A_{J}(r)$. The current density $\mathbf{j}$ is related to $\mathbf{A}$ via the London equation, $\mathbf{j}=-\mathbf{A}_{s} / \mu_{0} \lambda^{2}$, where $\mathbf{A}_{s}$ is the superfluid velocity expressed in units of vector potential; here $\mathbf{A}_{s}=A_{s} \hat{\varphi}$ with

$$
A_{s}(r)=A(r)-\Phi_{f} / 2 \pi r .
$$

The second term on the right-hand side is due to the gradient of the phase of the complex superconducting order parameter. For a ring with a slit we treat $\Phi_{f}$ as a free parameter to be determined by boundary conditions, but for an unslitted ring $\Phi_{f}$ corresponds to the London

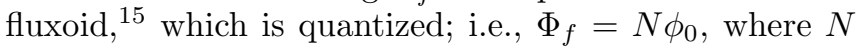
is an integer and $\phi_{0}=h / 2 e$ is the superconducting flux quantum.

It is useful to divide the fields into two contributions, $\mathbf{B}=\mathbf{B}_{1}+\mathbf{B}_{2}, A=A_{1}+A_{2}, \mathbf{j}=\mathbf{j}_{1}+\mathbf{j}_{2}, J=J_{1}+J_{2}$, etc., where the subscript $n=1$ indicates that it is driven by the fluxoid [driving term $D_{1}(r)=-\Phi_{f} / 2 \pi r$ and $\left.A_{1}(r)=A_{J 1}(r)\right]$, and the subscript $n=2$ indicates that it is driven by the applied field [driving term $D_{2}(r)=A_{a}(r)$ and $\left.A_{2}(r)=A_{a}(r)+A_{J 2}(r)\right]$. The London equation for contribution $n$ is

$$
J_{n}(r)=-\left[D_{n}(r)+A_{J n}(r)\right] / \mu_{0} \Lambda .
$$

In this paper we will calculate the two contributions $(n$ $=1$ and 2) to the magnetic flux $\Phi_{n}(a)$ through the hole, total flux $\Phi_{n}(b)$ through the ring, magnetic moment $m_{n}$, and total current $I_{n}$ around the ring using the definitions

$$
\begin{gathered}
\Phi_{n}(r)=2 \pi \int_{0}^{r} d r^{\prime} r^{\prime} B_{n}\left(r^{\prime}\right)=2 \pi r A_{n}(r), \\
m_{n}=\pi \int_{a}^{b} d r r^{2} J_{n}(r), \quad I_{n}=\int_{a}^{b} d r J_{n}(r) .
\end{gathered}
$$

The relation between the sheet current $J_{n}(r)$ and the vector potential $A_{J n}(r)$ it generates is obtained as follows. From the Maxwell equation $\mu_{0} \mathbf{j}=\nabla \times \mathbf{B}=$ $\nabla \times \nabla \times \mathbf{A}=-\nabla^{2} \mathbf{A}$ (since here $\nabla \cdot \mathbf{A}=0$ ) we obtain the 3D Biot-Savart law for the current-generated part $\mathbf{A}_{J}(\mathbf{r})$ of $\mathbf{A}$ :

$$
\mathbf{A}_{J}(\mathbf{r})=\mu_{0} \int d^{3} r^{\prime} \frac{\mathbf{j}\left(\mathbf{r}^{\prime}\right)}{4 \pi\left|\mathbf{r}-\mathbf{r}^{\prime}\right|}
$$

with $\mathbf{r}=(x, y, z)$. Integrating this over $z^{\prime}$ and $\varphi$, noting that $\mathbf{j}$ flows only inside the film $-d / 2 \leq z \leq d / 2$, one obtains in the plane $z=0$ for each component:

$$
A_{J n}(r)=\mu_{0} \int_{a}^{b} d r^{\prime} J_{n}\left(r^{\prime}\right) Q\left(r, r^{\prime}\right)
$$

with the integral kernel

$$
Q\left(r, r^{\prime}\right)=\int_{0}^{\pi} \frac{d \varphi}{2 \pi} \frac{r^{\prime} \cos \varphi}{\left(r^{2}+r^{\prime 2}-2 r r^{\prime} \cos \varphi\right)^{1 / 2}} .
$$

This kernel may be written in terms of elliptic integrals, but for transparency we prefer a fast direct numerical integration of (7); see also Refs. 23,45. High accuracy is achieved by substituting in the integral $\varphi=$ $\varphi(u)=\pi u-\sin (\pi u)$ and integrating over $0 \leq u \leq 1$ using an equidistant grid for $u, u_{i}=(i-1 / 2) / N_{\varphi}$, $i=1,2, \ldots, N_{\varphi}, N_{\varphi} \approx 30-60$, with weights $w_{i}=$ $\varphi^{\prime}(u) / N_{\varphi}=\left[1-\cos \left(\pi u_{i}\right)\right] \pi / N_{\varphi}$ :

$$
\begin{aligned}
Q\left(r, r^{\prime}\right)=\int_{0}^{\pi} d \varphi f(\varphi)= & \int_{0}^{1} d u f[\varphi(u)] \varphi^{\prime}(u) \\
& \approx \sum_{i=1}^{N_{\varphi}} f\left[\varphi\left(u_{i}\right)\right] w_{i} .
\end{aligned}
$$

Writing $J_{n}(r)=\int d r^{\prime} J_{n}\left(r^{\prime}\right) \delta\left(r-r^{\prime}\right)$ and inserting Eq. (2) into Eq. (6), we obtain

$$
D_{n}(r)=-\mu_{0} \int_{a}^{b} d r^{\prime} J_{n}\left(r^{\prime}\right)\left[Q\left(r, r^{\prime}\right)+\Lambda \delta\left(r-r^{\prime}\right)\right] .
$$

(For introduction of finite $\lambda$ into other geometries see Ref. 16.) Formally, the integral equation (9) may be solved for the sheet current $J(r)$ by writing

$$
J_{n}(r)=-\mu_{0}^{-1} \int_{a}^{b} d r^{\prime} D_{n}\left(r^{\prime}\right) K\left(r, r^{\prime}\right),
$$

where $K\left(r, r^{\prime}\right)$ is the inverse of the kernel $Q\left(r, r^{\prime}\right)+\Lambda \delta(r-$ $\left.r^{\prime}\right)$, defined by

$$
\int_{a}^{b} d r^{\prime} K\left(r, r^{\prime}\right)\left[Q\left(r^{\prime}, r^{\prime \prime}\right)+\Lambda \delta\left(r^{\prime}-r^{\prime \prime}\right)\right]=\delta\left(r-r^{\prime \prime}\right) .
$$

The inverse kernel $K\left(r, r^{\prime}\right)$ is easily calculated numerically by introducing an appropriate grid $r_{i}$ with weights $w_{i}$ such that the integral is approximated by a sum,

$$
\int_{a}^{b} d r f(r) \approx \sum_{i=1}^{N_{r}} f\left(r_{i}\right) w_{i} .
$$

High accuracy is achieved in the present case, where the integrated function $f(r)$ may have infinities at $r=a$ and $r=b$, by a grid that is very dense near $r=a$ and $r=b$. A good such grid is found by the substitution $r=r(u)=a+(b-a)\left(10 u^{2}-15 u^{3}+6 u^{5}\right), r^{\prime}(u)=30(b-$ a) $\left(u-u^{2}\right)^{2}$, yielding $r_{i}=r\left(u_{i}\right), w_{i}=r^{\prime}\left(u_{i}\right) / N_{r}$, with $u_{i}=(i-1 / 2) / N_{r}, i=1,2, \ldots, N_{r}, N_{r} \approx 30-100$. This grid defines the vectors $J_{n i}=J_{n}\left(r_{i}\right), D_{n i}=D_{n}\left(r_{i}\right)$, and the matrix $Q_{i j}=Q\left(r_{i}, r_{j}\right)$. Equation (9) then becomes a sum (or matrix multiplication):

$$
D_{n i}=-\sum_{j=1}^{N_{r}}\left(w_{i} Q_{i j}+\Lambda \delta_{i j}\right) \mu_{0} J_{n j} .
$$

This is inverted by

$$
\mu_{0} J_{n i}=-\sum_{j=1}^{N_{r}} K_{i j} D_{n j},
$$


where $K_{i j}$ is an inverse matrix:

$$
K_{i j}=\left(w_{i} Q_{i j}+\Lambda \delta_{i j}\right)^{-1}
$$

(no summation over $i ; \delta_{i j}=1$ if $i=j$; otherwise $\delta_{i j}=0$ ). The matrix equation from which the total sheet current is determined for given $B_{a}$ and $\Phi_{f}$ thus reads explicitly:

$$
\mu_{0} J\left(r_{i}\right)=-\sum_{j=1}^{N_{r}} K_{i j}\left(\frac{r_{j}}{2} B_{a}-\frac{\Phi_{f}}{2 \pi r_{j}}\right) .
$$

Finally, one hint is required without which this method may not work or is inaccurate. The matrix $Q_{i j}$ has infinite diagonal terms $Q_{i i}$, since $Q\left(r, r^{\prime}\right)$, Eq. (7), diverges logarithmically when $r$ approaches $r^{\prime}$. Namely, one has

$$
Q_{i, i+1} \approx Q\left(r_{i}, r_{i}+w_{i}\right) \approx \frac{1}{2 \pi} \ln \frac{r_{i}}{w_{i}} .
$$

This problem was dealt with in detail in Refs. 2 and 3, where the optimum choice of the $Q_{i i}$ was found first numerically ${ }^{2}$ and then analytically ${ }^{3}$, e.g., from the condition that an infinite disk ideally screens two coils separated by the disk. One thus has the complete definition of the matrix $Q_{i j}$ :

$$
\begin{aligned}
Q_{i j} & =Q\left(r_{i}, r_{j}\right), \quad i \neq j, \\
Q_{i i} & =\frac{1}{2 \pi}\left(\ln \frac{16 \pi r_{i}}{w_{i}}-2\right) .
\end{aligned}
$$

In Eq. (15) the diagonal term is $w_{i} Q_{i i}+\Lambda$. Thus, when $\Lambda$ is larger than the maximum value of the weight $w_{i}$ (or the spacing between grid points), which is of order $(b-a) / N_{r}$, then the choice of the $Q_{i i}$ is not critical, and for the computation of $J(r)$ one may even put $Q_{i i}=0$. For small $\Lambda$, however, the correct choice of $Q_{i i}$ is important.

\section{SELF-INDUCTANCE OF FLAT RINGS}

According to Eq. (16) the current in a ring originates from either an applied field $B_{a}$, trapped flux related to the parameter $\Phi_{f}$, or both. In this section we put $B_{a}=0$ and compute the sheet current $J_{1}(r)$ for finite trapped fluxoid $\Phi_{f}$. The total current $I_{1}$ is then given by Eq. (4). The current generates a flux $\Phi_{1}(r)=2 \pi r A_{1}(r)$, Eq. (3), with $A_{1}(r)=A_{J 1}(r)$ from Eq. (6). Figure 1 shows the profiles of the sheet current $J_{1}(r)$, perpendicular induction $B_{1}(r)=(1 / r)\left(r A_{1}\right)^{\prime}$, and vector potential $A_{1}(r)$ for a ring with $a / b=0.4$ for three $\Lambda / b$ values $(0,0.03,0.1)$ for the case of flux trapping with $B_{a}=0$ and $\Phi_{f}>0$. One can see that finite $\Lambda$ removes the infinities of $J_{1}(r)$ at $r=a$ and $r=b\left[J_{1} \propto(r-a)^{-1 / 2}\right.$ and $J_{1} \propto(b-r)^{-1 / 2}$; see Ref. 1], which lead to a similar infinity of $B_{1}(r)$. For $\Lambda>0, J_{1}(a)$ and $J_{1}(b)$ are finite, and $B_{1}(r)$ penetrates the superconductor but still exhibits a logarithmic infinity at $r=a$ and $r=b$, which is caused by the abrupt jump of $J_{1}(r)$ to zero. Note also that with increasing $\Lambda$ the total current $I_{1}$, the magnetic flux $\Phi_{1}(a)$ in the hole,

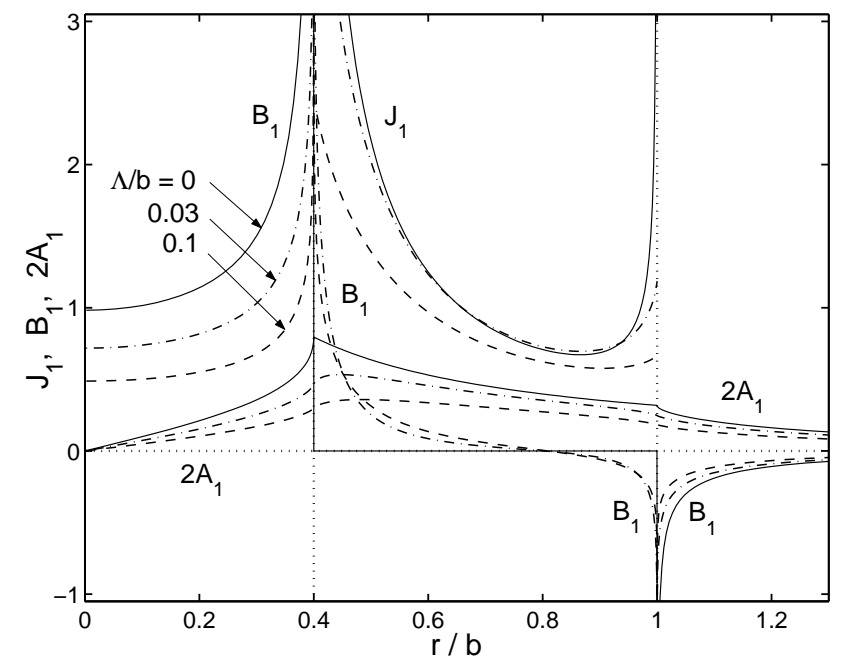

FIG. 1: Profiles of sheet current $J_{1}(r)$ in the ring, perpendicular magnetic induction $B_{1}(r)=(1 / r)\left(r A_{1}\right)^{\prime}$, and currentgenerated vector potential $A_{1}(r)=A_{J 1}(r)$ in the plane of the ring for the case of trapped flux and zero applied field $\left(B_{a}=0\right.$, $\Phi_{f}>0$, Figs. 1-6). The hole radius is $a=0.4 b$ and the 2D penetration depth is $\Lambda=0$ (solid curves), $\Lambda=0.03 b$ (dotdashed), and $\Lambda=0.1 b$ (dashed). Plotted are the dimensionless quantities $J_{1} /\left(\Phi_{f} / \mu_{0} b^{2}\right), B_{1} /\left(\Phi_{f} / b^{2}\right)$, and $2 A_{1} /\left(\Phi_{f} / b\right)$.

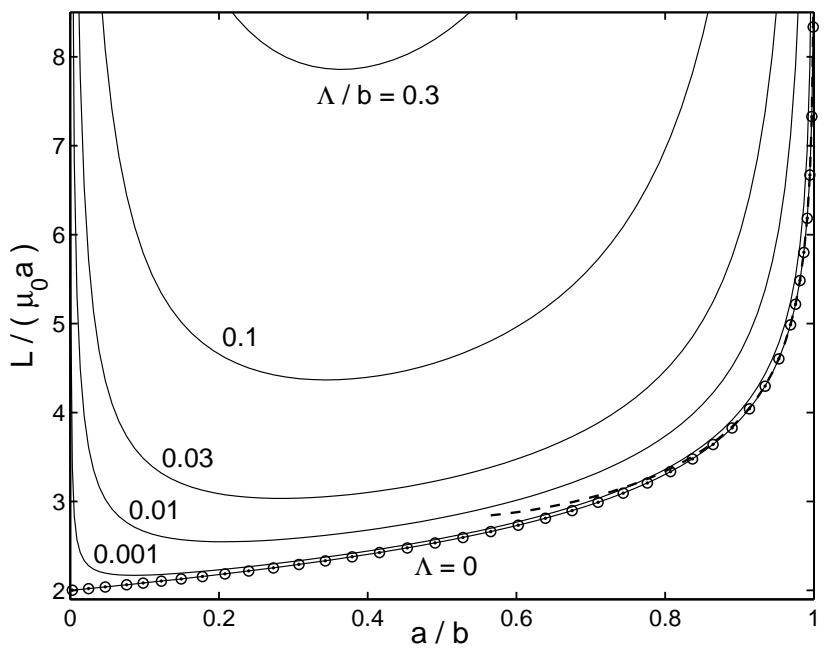

FIG. 2: Computed self-inductance $L=\Phi_{f} / I_{1}$ of a superconducting thin flat ring with hole radius $a$ and outer radius $b$, plotted in units of $\mu_{0} a$ for $2 \mathrm{D}$ penetration depths $\Lambda=0.001$, $0.01,0.03,0.1$, and 0.3 in units of $b$. Note that even very small $\Lambda \ll b$ considerably enhances the inductance of rings with a small central hole $(a / b \ll 1)$ as compared with the case $\Lambda=0$. The circles depict the empirical expression (20) valid for $\Lambda=0$; they perfectly coincide with the dots that mark the curve computed for $\Lambda=0$. The dashed curve shows the limiting expression $L_{1}$, Eq. (19). See also Fig. 6 below, showing the inverse self-inductance $\mu_{0} a / L$. 


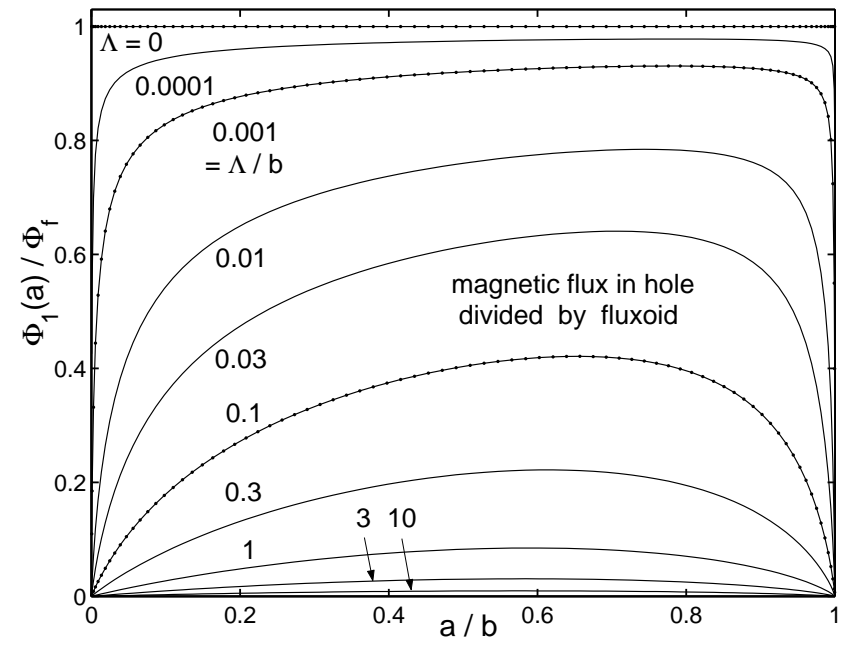

FIG. 3: Magnetic flux $\Phi_{1}(a)$ in the hole of a superconducting thin flat ring with hole radius $a$ and outer radius $b$ for the trapped-flux case as in Figs. 1-6 (applied field $B_{a}=0$ and fluxoid $\Phi_{f}>0$ ) for $2 \mathrm{D}$ penetration depths $\Lambda / b=0,0.0001$, $0.001,0.01,0.03,0.1,0.3,1,3$, and 10 . The dots on some of the curves mark the $a / b$ grid used here in all such figures.

and the field $B_{1}$ in the hole decrease, while the London fluxoid $2 \pi r\left[A_{1}(r)+\mu_{0} \Lambda J_{1}(r)\right]=\Phi_{f}$ remains constant 17

In the case of ideal screening, i.e. for $\Lambda=0$, no magnetic field penetrates the ring material, and thus the flux $\Phi_{1}(r)$ is the same for any $r$ between $r=a$ and $r=b$. The self-inductance of the ring may then be defined as $L=\Phi_{1} / I_{1}$ with $\Phi_{1}=\Phi_{1}(a)=\Phi_{1}(b)$. For small reduced inner radius $\tilde{a} \equiv a / b \ll 1$ the flux trapped in the hole was found by Clem ${ }^{1.11}$ to be $\Phi_{1}(a)=2 \mu_{0} a I_{1}$; thus the inductance $L=\Phi_{1} / I_{1}$ approaches $L_{0}=2 \mu_{0} a$. In the opposite limit, for narrow rings with $\tilde{a} \rightarrow 1, L$ approaches ${ }^{1.5}$

$$
\begin{aligned}
L_{1} & =\mu_{0} R[\ln (8 R / w)-2+\ln 4] \\
& =\mu_{0} b(1+\tilde{a})\left(\tanh ^{-1} \tilde{a}-1+\ln 4\right)
\end{aligned}
$$

$[R=(a+b) / 2, w=b-a]$. For arbitrary $\tilde{a}$, but still $\Lambda=0, L$ was computed in Ref. 1 , where a useful empirical formula was presented,

$$
L_{2}=\mu_{0} b\left[\tilde{a}-0.197 \tilde{a}^{2}-0.031 \tilde{a}^{6}+(1+\tilde{a}) \tanh ^{-1} \tilde{a}\right] .
$$

This fit is confirmed by our method, as shown in Fig. 2; its relative deviation from the exact $L$ ranges from $-0.005 \%$ to $+0.06 \%$.

When the effective penetration depth $\Lambda$ is finite, the magnetic field penetrates into the ring material, and the magnetic flux $\Phi_{1}(r)$ is no longer constant when $r$ changes from $r=a$ to $r=b$. The definition of $L=\Phi_{1} / I_{1}$ via magnetic flux is thus not unique; in particular, $\Phi_{1}(a) / I_{1} \neq \Phi_{1}(b) / I_{1}$ when $\Lambda>0$. In this general case one must use the definition of $L$ via the electromagnetic energy of the ring, $E=(1 / 2) L I_{1}^{2}$. This energy is composed of the magnetic energy $E_{m}$ and the kinetic energy $E_{k}$ of the currents, as is evident from the energy integral

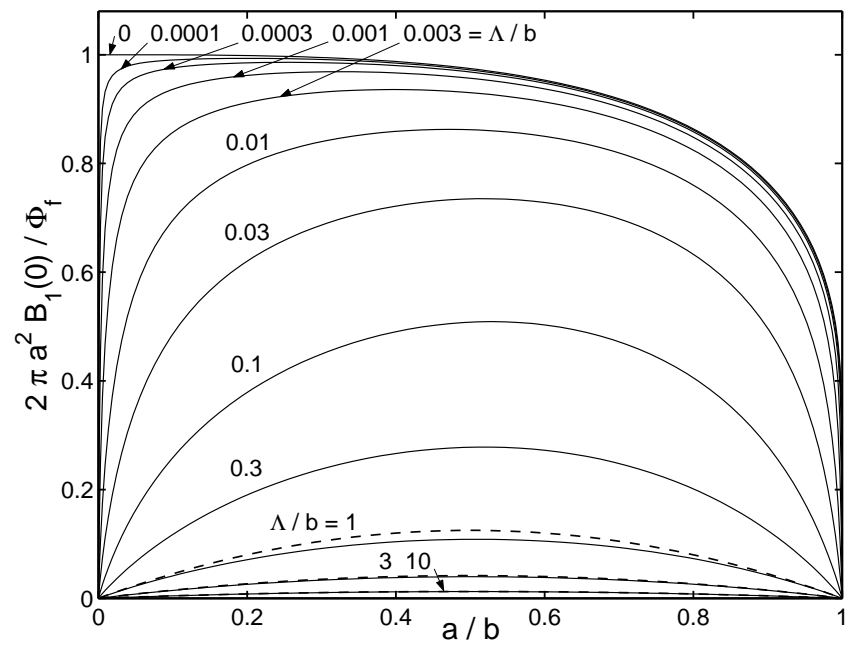

FIG. 4: Minimum of the magnetic field $B_{1}(0)$ occurring in the center of the ring for the trapped-flux case $\left(B_{a}=0\right.$ and $\left.\Phi_{f}>0\right)$ plotted as $2 \pi a^{2} B_{1}(0) / \Phi_{f}$ for $\Lambda / b=0,0.0001,0.0003$, $0.001,0.003,0.01,0.03,0.1,0.3,1,3$, and 10. The dashed curves show the large- $\Lambda$ approximation, Eq. (41), for $\Lambda / b=1$, 3,10 .

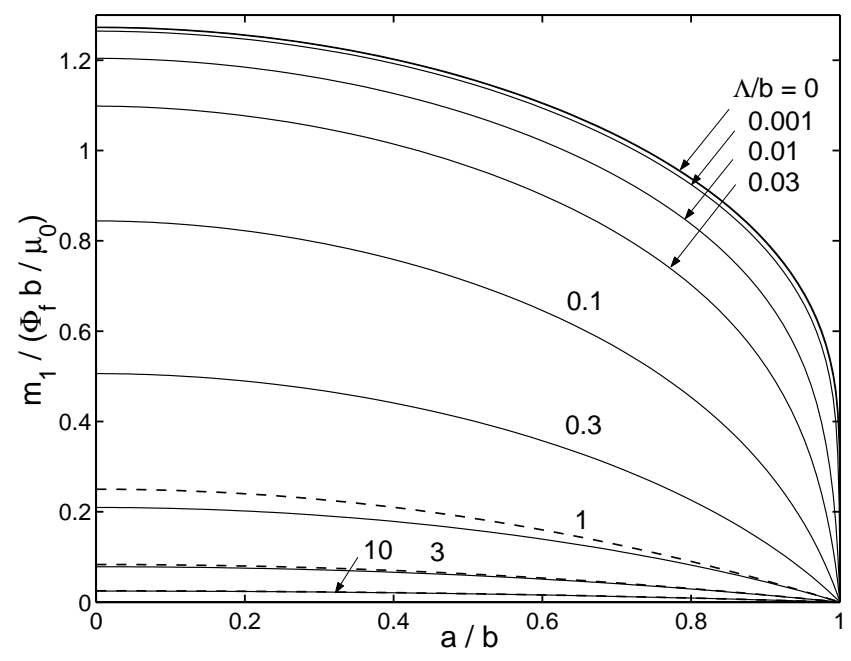

FIG. 5: Magnetic moment $m_{1}$ of a superconducting thin flat ring with inner and outer radii $a$ and $b$ for the trapped-flux case $\left(B_{a}=0\right.$ and $\left.\Phi_{f}>0\right)$ plotted as the dimensionless ratio $\alpha_{m}=\mu_{0} m_{1} / b \Phi_{f}$ for $\Lambda / b=0,0.001,0.01,0.03,0.1,0.3,1,3$, and 10. The dashed curves show the large- $\Lambda$ approximation, Eq. (42), for $\Lambda / b=1,3,10$. See also Fig. 12 below.

of London theory:

$$
E=E_{m}+E_{k}=\frac{\mu_{0}}{2} \int d^{3} r\left(\mathbf{H}_{1}^{2}+\lambda^{2} \mathbf{j}_{1}^{2}\right)
$$

with $\mathbf{H}_{1}=\mu_{0}^{-1} \mathbf{B}_{1}$ and $\mathbf{j}_{1}=\nabla \times \mathbf{H}_{1}$. The integral (21) over all space can be transformed into an integral over the superconductor by introducing the vector potential. For a flat ring this yields

$$
E=E_{m}+E_{k}=\pi \int_{a}^{b} d r r\left[J_{1}(r) A_{1}(r)+\mu_{0} \Lambda J_{1}(r)^{2}\right]
$$




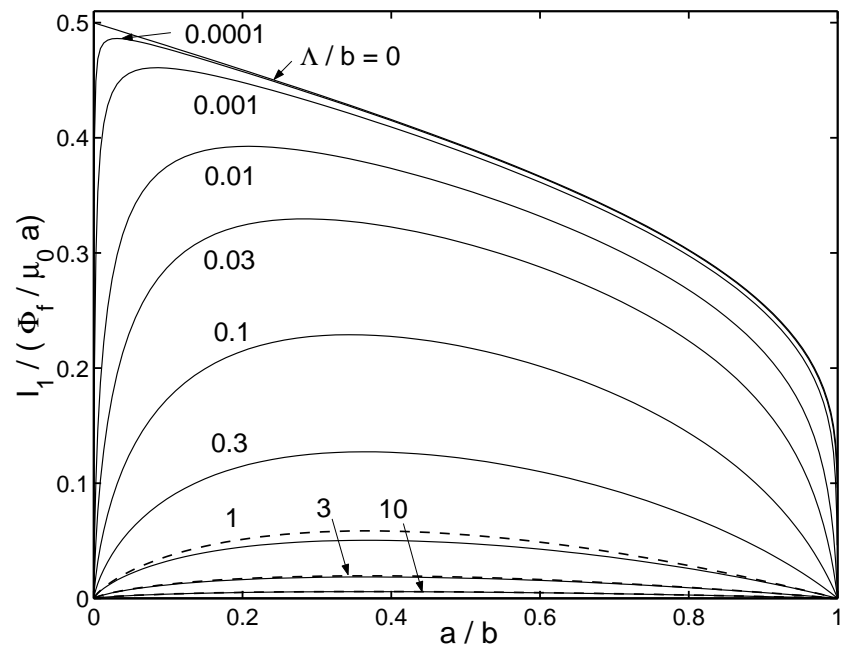

FIG. 6: Total current $I_{1}$ in the flat ring with trapped flux $\left(B_{a}=0\right.$ and $\left.\Phi_{f}>0\right)$ plotted as the dimensionless ratio $\alpha_{I}=$ $\mu_{0} a I_{1} / \Phi_{f}=\mu_{0} a / L$ for $\Lambda / b=0,0.0001,0.001,0.01,0.03,0.1$, $0.3,1,3$, and 10. Same data as in Fig. 2 but inverted. The dashed curves show the large- $\Lambda$ approximation, Eq. (40), for $\Lambda / b=1,3,10$.

From the two energy terms when $B_{a}=0$ one may define the geometric inductance $L_{m}=2 E_{m} / I_{1}^{2}$ and the kinetic inductance $L_{k}=2 E_{k} / I_{1}^{2}$, yielding the total selfinductance $L=L_{m}+L_{k}=2 E / I_{1}^{2}$. The energy $E$, Eq. (22), may also be written as

$$
E=E_{m}+E_{k}=(1 / 2) \Phi_{f} I_{1},
$$

where $\Phi_{f}=2 \pi r\left(A_{1}+\mu_{0} \Lambda J_{1}\right)=$ const (for $a \leq r \leq b$ ) is the London fluxoid, ${ }^{15.17}$ We may derive Eq. (23) from (22) by noting that the constant combination $2 \pi r\left(A_{1}+\right.$ $\left.\mu_{0} \Lambda J_{1}\right)=\Phi_{f}$ can be factored out of the integrand.

From Eq. (23) and the definition $E=(1 / 2) L I_{1}^{2}$ it immediately follows that

$$
L=\Phi_{f} / I_{1}
$$

This general result, valid for arbitrary penetration depth $\Lambda$, differs from the definition used previously ${ }^{1}$ for $\Lambda=$ 0 , in that the magnetic flux $\Phi_{1}(a)$ through the hole is replaced by the fluxoid $\Phi_{f}$, which coincides with the flux $\Phi_{1}(a)$ in the hole only in the special case $\Lambda=0$.

Equation (23) also may be derived by considering how the fluxoid in the ring may be increased from zero to $\Phi_{f}$ by moving vortices (Pearl vortices ${ }^{18.19 .20}$ of short length d) from the outer radius through the ring into the hole. Each vortex has to cross the current-carrying ring, where a Lorentz force $\phi_{0} J_{1}(r)$ acts on it. Integrating this force from $r=b$ to $r=a$, one obtains the energy $\phi_{0} I_{1}$. Each crossing vortex increases the phase change of the superconductor order parameter around a circle in the ring by $2 \pi$ and thus increases the fluxoid by $\phi_{0}$. Noting that the total current $I_{1}$ is proportional to the number of vortices that already are in the hole, one obtains $E=(1 / 2) \Phi_{f} I_{1}$, Eq. (23).

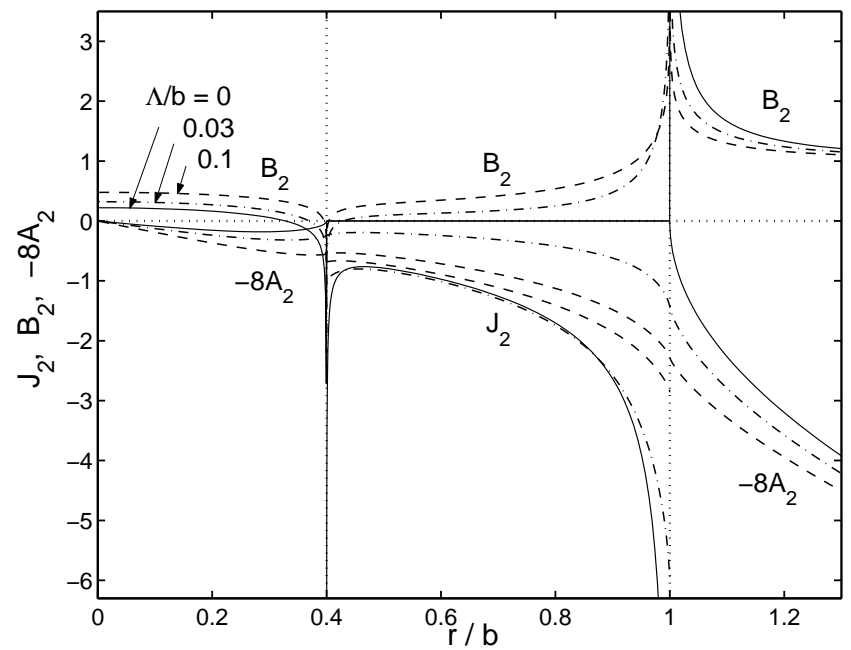

FIG. 7: Profiles of sheet current $J_{2}(r)$ in the ring, perpendicular magnetic induction $B_{2}(r)=(1 / r)\left(r A_{2}\right)^{\prime}$, and vector potential $A_{2}(r)$ in the plane of the ring for the zero-fluxoid state in a finite applied field $\left(B_{a}>0, \Phi_{f}=0\right.$, Figs. 7-12). The hole radius is $a=0.4 b$ and the $2 \mathrm{D}$ penetration depth is $\Lambda=0$ (solid curves), $\Lambda=0.03 b$ (dot-dashed), or $\Lambda=0.1 b$ (dashed), as in Fig. 1. Plotted are the dimensionless quantities $J_{2} / H_{a}, B_{2} / B_{a}$, and $-8 A_{2} / b B_{a}$.

Figure 2 shows the inductance $L=\Phi_{f} / I_{1}$ for various ratios $\Lambda / b=0,0.001,0.01,0.03,0.1$, and 0.3 . The circles show the fit (20), which is an excellent approximation for $\Lambda=0$ and all hole radii $a$. Note that $L$ increases with increasing penetration depth $\Lambda$. Even small $\Lambda / b=$ 0.001 noticeably enhances $L$ of rings with a small hole. Figure 3 shows the magnetic flux in the hole, $\Phi_{1}(a)=$ $2 \pi a A_{1}(a)$, referred to the fluxoid $\Phi_{f}$ trapped in the ring. Note that $\Phi_{1}(a)$ may be much smaller than $\Phi_{f}$ even for small $\Lambda / b$. A similar plot, Fig. 4, shows the minimum field in the hole, $B_{1}(0)$, occurring at the center $r=0$ (cf. Fig. 1) plotted as $2 \pi a^{2} B_{1}(0) / \Phi_{f}$. These curves look qualitatively similar to $\Phi_{1}(a) / \Phi_{f}$ in Fig. 3. For $\Lambda=0$ and $a \ll b$ one has $2 \pi a^{2} B_{1}(0) / \Phi_{f} \rightarrow 1$.

The magnetic moment $m_{1}$ of this ring (still for $B_{a}=0$ and $\left.\Phi_{f}>0\right)$ is depicted in Fig. 5 as the dimensionless ratio $\alpha_{m}=\mu_{0} m_{1} / b \Phi_{f}$; for further approximations to these curves see the dotted and dot-dashed curves in the similar Fig. 12 below. The total current $I_{1}$ times the inner radius $a$ is depicted in Fig. 6 as the dimensionless ratio $\alpha_{I}=\mu_{0} a I_{1} / \Phi_{f}$. Actually Fig. 6 shows the same data as Fig. 2, but inverted, since the plotted quantity $\mu_{0} a I_{1} / \Phi_{f}$ equals $\mu_{0} a / L$; however, Fig. 6 shows the entire range $0 \leq \Lambda \leq \infty$.

\section{ZERO-FLUXOID STATE}

This section considers the zero-fluxoid state reached when a thin superconducting ring is slowly cooled in zero field. A magnetic field $B_{a}=\mu_{0} H_{a}$ is then applied, but the fluxoid $\Phi_{f}$ in the ring then remains zero. When $\Lambda=$ 

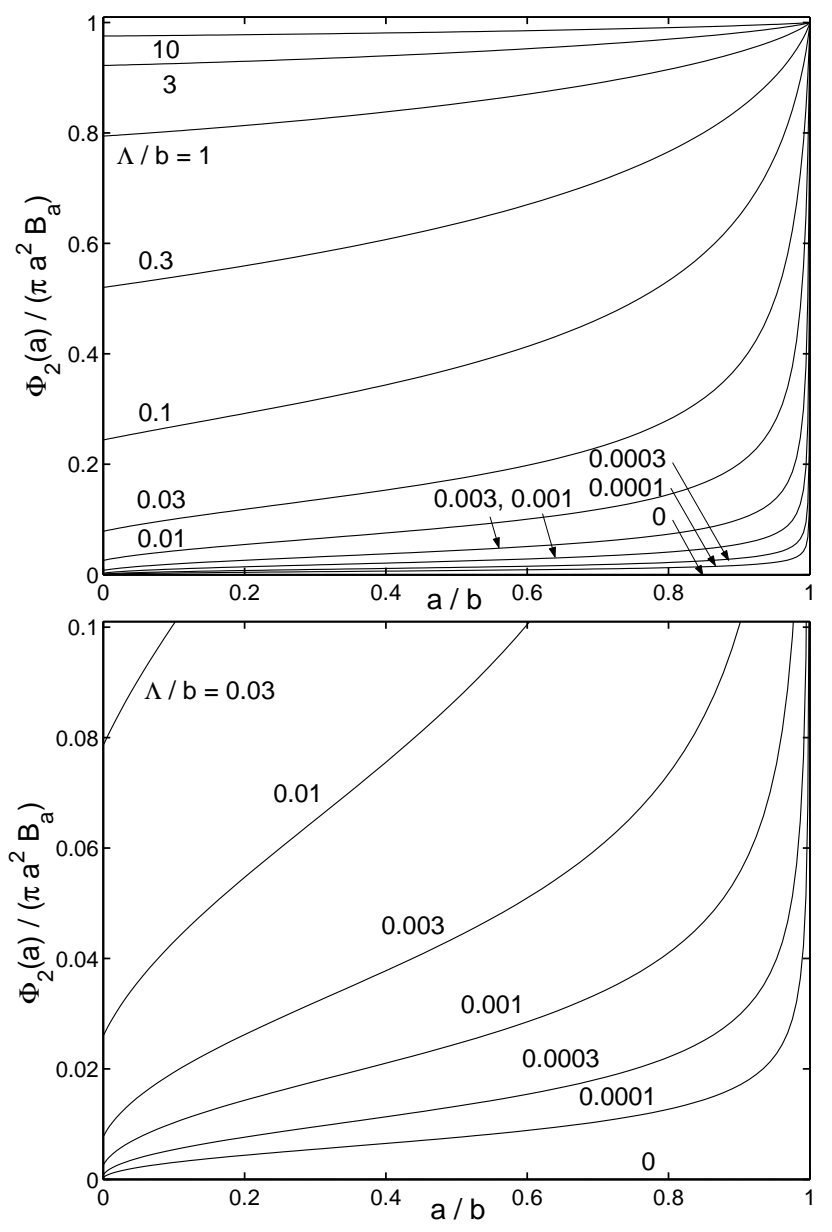

FIG. 8: Magnetic flux $\Phi_{2}(a)$ in the hole of a flat ring when $B_{a}>0$ and $\Phi_{f}=0$ in units of $\pi a^{2} B_{a}$ for $\Lambda / b=0,0.0001$, $0.0003,0.001,0.003,0.01,0.03,0.1,0.3,1,3$, and 10 . The bottom plot shows the same data ten times enlarged.

0 , one has ideal screening, such that the magnetic flux $\Phi(a)$ in the hole is zero. For finite $\Lambda>0$ the screening is incomplete, some flux leaks through the ring, and the flux $\Phi(a)$ in the hole is no longer zero. In this section we therefore put $B_{a}>0$ and $\Phi_{f}=0$ in our numerics. Figure 7 shows for this case the profiles of $J_{2}(r), B_{2}(r)$, and $A_{2}(r)$ for a ring with $a / b=0.4$ for $\Lambda / b=0,0.03$, and 0.1 . Note that the induction $B_{2}(r)$ changes sign inside the hole and has a negative infinity at the inner edge $r=a$ and a positive infinity at the outer edge $r=b$. For $\Lambda=0$ the integral of $B_{2}(r)$ over the hole area is $\Phi_{2}(a)=0$, but for $\Lambda>0$ the flux $\Phi_{2}(a)>0$.

Figure 8 shows the penetrated flux $\Phi_{2}(a)$ in units of its maximum value $\pi a^{2} B_{a}$ reached in the limit of $\Lambda \gg b$, and Fig. 9 shows the field maximum $B_{2}(0)$ occurring at the center $r=0$ of the ring. Both $\Phi_{2}(a)$ and $B_{2}(0)$ increase monotonically with both $a$ and $\Lambda$, but while $B_{2}(0)$ at small $a$ increases linearly with the radius $a$, the penetrated flux $\Phi_{2}(a)$ at small $a$ and small $\Lambda$ has negative curvature; see bottom of Fig. 8 . The penetrated flux $\Phi_{2}(a) / \pi a^{2} B_{a} \approx B_{2}(0) / B_{a}$ in the limit of small hole ra-

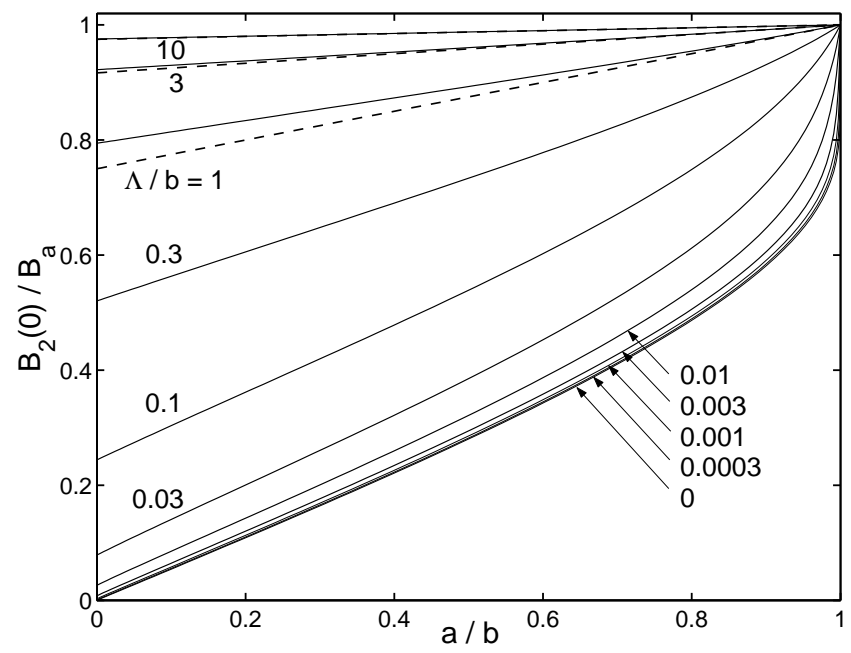

FIG. 9: Magnetic field $B_{2}(0)$ at the center $r=0$ of a thin flat ring when $B_{a}>0$ and $\Phi_{f}=0$ in units $B_{a}$ for $\Lambda / b=0$, $0.0003,0.001,0.003,0.01,0.03,0.1,0.3,1,3$, and 10 . The dashed curves show the large- $\Lambda$ approximation, Eq. (43), for $\Lambda / b=1,3,10$.

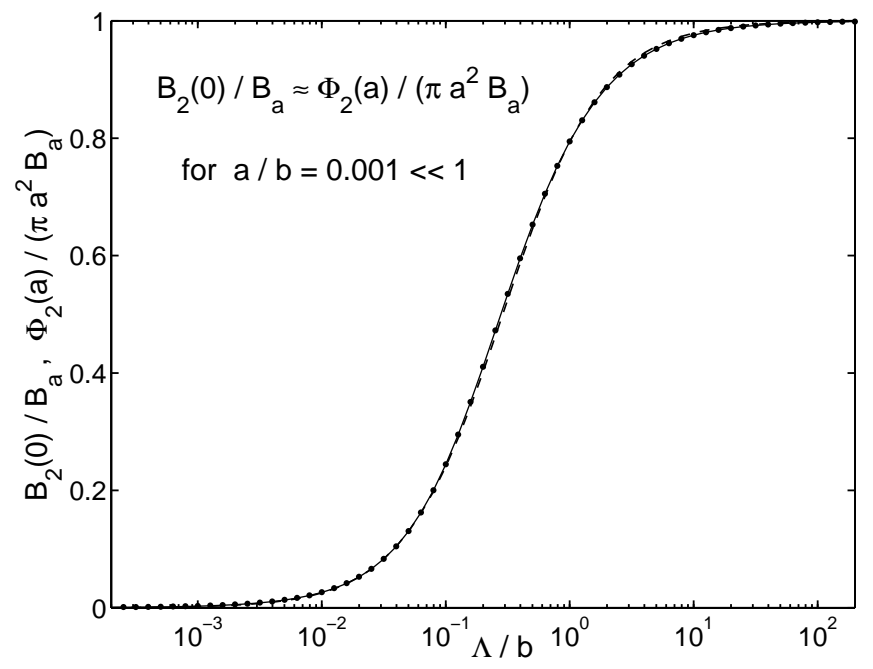

FIG. 10: Penetrated flux in thin flat rings with small hole radius $a \ll b$ for $B_{a}>0, \Phi_{f}=0$, plotted versus $\Lambda / b$ as $\Phi_{2}(a) / \pi a^{2} B_{a}$ (dots), which in this limit is nearly equal to $B_{2}(0) / B_{a}$ (solid curve) and is well approximated by Eq. (25), shown as the dashed curve.

dius $a / b \ll 1$ is depicted in Fig. 10 as a function of $\Lambda / b$. This curve is well fitted by

$$
\frac{\Phi_{2}(a)}{\pi a^{2} B_{a}} \approx \frac{B_{2}(0)}{B_{a}} \approx \frac{1}{2}+\frac{1}{2} \tanh \left(2.88 \ln \frac{\Lambda}{b}-0.675\right) .
$$

Figure 11 shows the magnetic moment $m_{2}$ of the ring in units of $m_{0}=-(8 / 3) b^{3} H_{a}$, which is reached for ideally screening disks $(a=\Lambda=0) ; 2.21$ the dimensionless ratio plotted is $\beta_{m}=m / m_{0}$. For $\Lambda=0$ and $a \rightarrow b$ one has the 


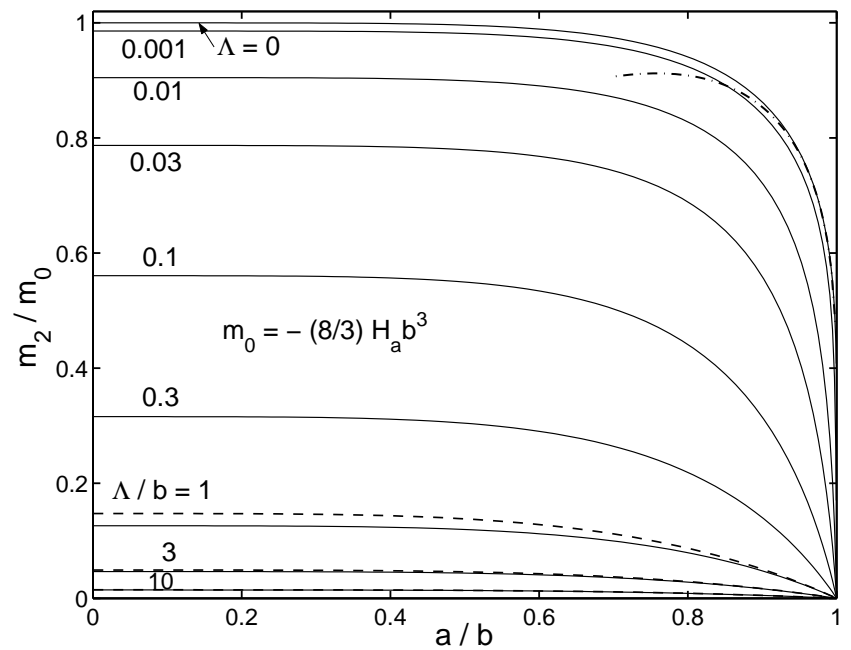

FIG. 11: Magnetic moment $m_{2}$ of a thin flat ring when $B_{a}>0$ and $\Phi_{f}=0$, plotted as the dimensionless ratio $\beta_{m}=m_{2} / m_{0}$, where $m_{0}=-(8 / 3) H_{a} b^{3}$ for $\Lambda / b=0,0.001$, $0.01,0.03,0.1,0.3,1,3$, and 10 . The dot-dashed curve shows the limit $b \rightarrow a$, Eq. (26). The dashed curves show the large- $\Lambda$ approximation, Eq. (44), for $\Lambda / b=1,3,10$.

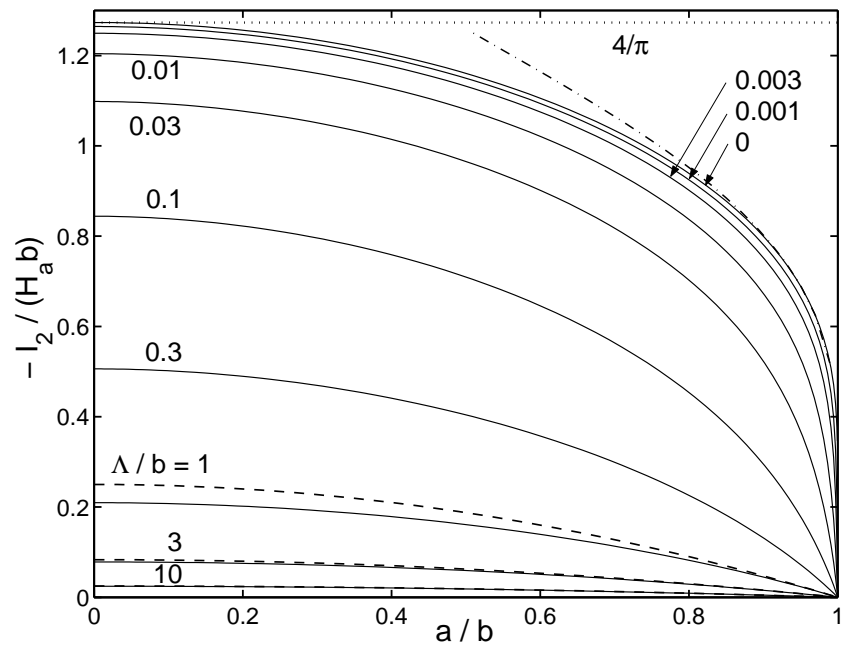

FIG. 12: Total current $I_{2}$ in a thin flat ring when $B_{a}>0$ and $\Phi_{f}=0$ for $\Lambda / b=0,0.001,0.003,0.01,0.03,0.1,0.3,1$, 3 , and 10, plotted as the dimensionless ratio $\beta_{I}=-I_{2} / b H_{a}$. The dotted and dot-dashed curves show the limits, Eq. (27). Note that the curves of $\beta_{I}=-I_{2} / b H_{a}$ agree exactly with those in Fig. $5, \alpha_{m}=\mu_{0} m_{1} / b \Phi_{f}$, although they describe different physical quantities. The dashed curves show the large- $\Lambda$ approximation, Eq. (45), for $\Lambda / b=1,3,10$.

$\operatorname{limit} t^{5}$

$$
\frac{m_{2}}{m_{0}}=\frac{3 \pi^{2}}{128} \frac{(1+a / b)^{3}}{\tanh ^{-1}(a / b)-1+\ln 4} .
$$

Figure 12 shows the total current $I_{2}$ in the ring induced by the applied field, expressed in terms of the dimensionless ratio $\beta_{I}=-I_{2} / b H_{a}$. The limits for $\Lambda=0$ are ${ }^{1.5}$

$$
I_{2}=\quad-(4 / \pi) b H_{a} \quad \text { for } a \rightarrow 0,
$$

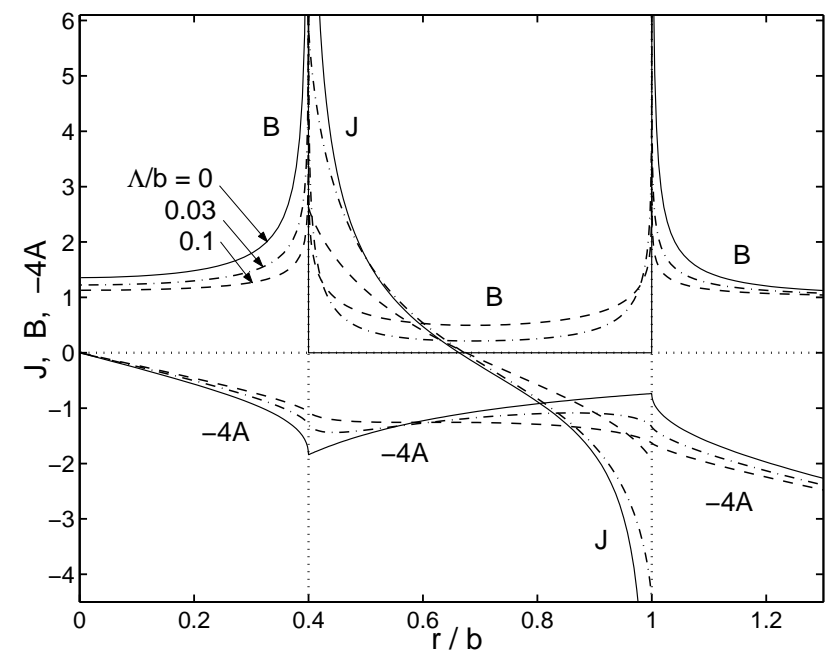

FIG. 13: Profiles of sheet current $J(r)$ in the ring, perpendicular magnetic induction $B(r)=(1 / r)(r A)^{\prime} \mathrm{m}$ and vector potential $A(r)$ in the plane of the ring for the case of zero total current $I=0$ (flux focusing, Figs. 13-17). The hole radius is $a=0.4 b$ and the $2 \mathrm{D}$ penetration depths are $\Lambda=0$ (solid curves), $\Lambda=0.03 b$ (dot-dashed), or $\Lambda=0.1 b$ (dashed), as in Figs. 1 and 7. Plotted are the dimensionless quantities $J / H_{a}$, $B / B_{a}$, and $-4 A / b B_{a}$.

$$
I_{2}=-\frac{(\pi / 4)(a+b) H_{a}}{\tanh ^{-1}(a / b)-1+\ln 4} \quad \text { for } a \rightarrow b
$$

Note that the curves in Fig. 12 exactly coincide with the curves in Fig. 5, even though they depict different physical quantities for different cases. This identity, $\alpha_{m}=\beta_{I}$, can be proved by evaluating the sum of energy integrals $F_{12}=\int d^{3} r \mathbf{B}_{1} \cdot \mathbf{B}_{2} / \mu_{0}+\int d^{3} r \mu_{0} \lambda^{2} \mathbf{j}_{1} \cdot \mathbf{j}_{2}$, where the first integral extends over all space, including the coil producing the applied field $B_{a}$, and the second integral extends only over the volume of the ring. With the help of the vector identity $\nabla \cdot(\mathbf{A} \times \mathbf{B})=\mathbf{B} \cdot \nabla \times \mathbf{A}-\mathbf{A} \cdot(\nabla \times \mathbf{B})$ with $\mathbf{A}=\mathbf{A}_{2}$ and $\mathbf{B}=\mathbf{B}_{1}$, the divergence theorem, and Eq. (2) with $n=2$, we can show that $F_{12}=0$. Then, using the same vector identity but with $\mathbf{A}=\mathbf{A}_{1}$ and $\mathbf{B}=\mathbf{B}_{2}$, the divergence theorem, Ampere's law, and Eqs. (2), (4), (6), and (7) with $n=1$, we obtain $F_{12}=m_{1} B_{a}+\Phi_{f} I_{2}=0$, which yields $\alpha_{m}=\mu_{0} m_{1} / b \Phi_{f}=-\mu_{0} I_{2} / b B_{a}=\beta_{I}$. This also can be proved by inserting $J_{1}$ and $J_{2}$ of Eq. (10) into the definitions (4) of $m_{1}$ and $I_{2}$, renaming the variables $r \leftrightarrow$ $r^{\prime}$, and noting the symmetry of $K\left(r, r^{\prime}\right)=K\left(r^{\prime}, r\right)\left(r^{\prime} / r\right)$ defined by Eq. (11). This symmetry follows from the symmetry of $Q\left(r, r^{\prime}\right)=Q\left(r^{\prime}, r\right)\left(r^{\prime} / r\right)$ defined by Eq. (7), from Eq. (11) noting that $\left(r^{\prime \prime} / r\right) \delta\left(r-r^{\prime \prime}\right)=\delta\left(r-r^{\prime \prime}\right)$, and from the additional property of the inverse kernel that $\int_{a}^{b} d r^{\prime}\left[Q\left(r^{\prime \prime}, r^{\prime}\right)+\Lambda \delta\left(r^{\prime \prime}-r^{\prime}\right)\right] K\left(r^{\prime}, r\right)=\delta\left(r^{\prime \prime}-r\right)$.

\section{FLUX FOCUSING}

This section considers a ring that is in a perpendicular magnetic field $B_{a}$ and contains a fluxoid $\Phi_{f}$ chosen such 


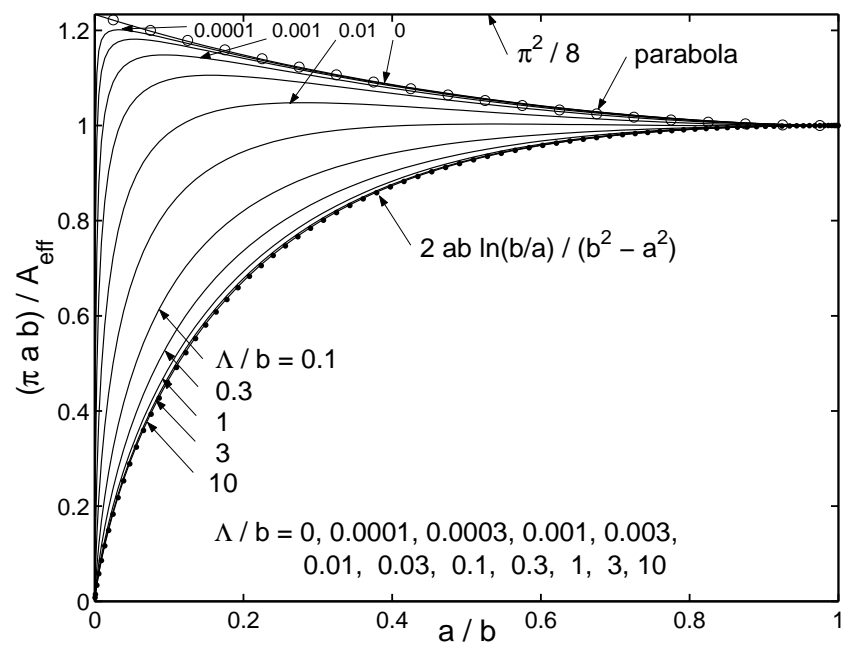

FIG. 14: Reciprocal of the effective area $A_{\text {eff }}=\Phi_{f} / B_{a}$ of a thin flat ring with $I=0$ (flux focusing) plotted as $(\pi a b) / A_{\text {eff }}$ versus the radius ratio $a / b$ for penetration depths $\Lambda / b=0$, $0.0001,0.0003,0.001,0.003,0.01,0.03,0.1,0.3,1,3$, and 10. The circles mark the parabolic fit, Eq. (29), and the dots nearly coinciding with the curves $\Lambda / b=3$ and 10 show the limit $\Lambda \rightarrow \infty$, Eqs. (30), (47).

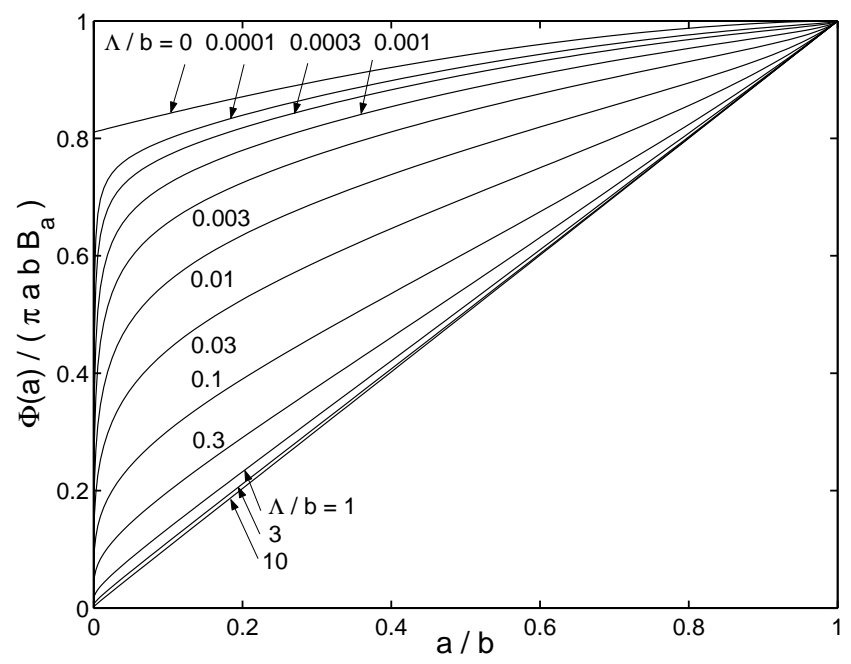

FIG. 15: Magnetic flux $\Phi(a)$ in the hole of a thin flat ring with $I=0$ (flux focusing) plotted as $\Phi(a) / \pi a b B_{a}$ versus $a / b$ for $\Lambda / b=0,0.0001,0.0003,0.001,0.003,0.01,0.03,0.1,0.3$, 1,3 , and 10 .

that no net current circulates around the ring. This circularly symmetric situation approximates a ring with a narrow slit along one of its radii as proposed by Clem.1.11 It neglects both the magnetic flux in the slit and the effects of the radial currents that flow in opposite directions alongside the slit. The slit interrupts the current I and allows magnetic flux to penetrate into the hole such that the condition $I=0$ holds. When $B_{a}$ is increased with ramp rate $d B_{a} / d t$, a voltage $U$ appears across the slit at radius $\mathrm{r}, U=-d \Phi_{f} / d t=-\left(d B_{a} / d t\right) A_{\text {eff }}$, where $A_{\text {eff }}=\Phi_{f} / B_{a}$ is the effective area of the slitted ring. In

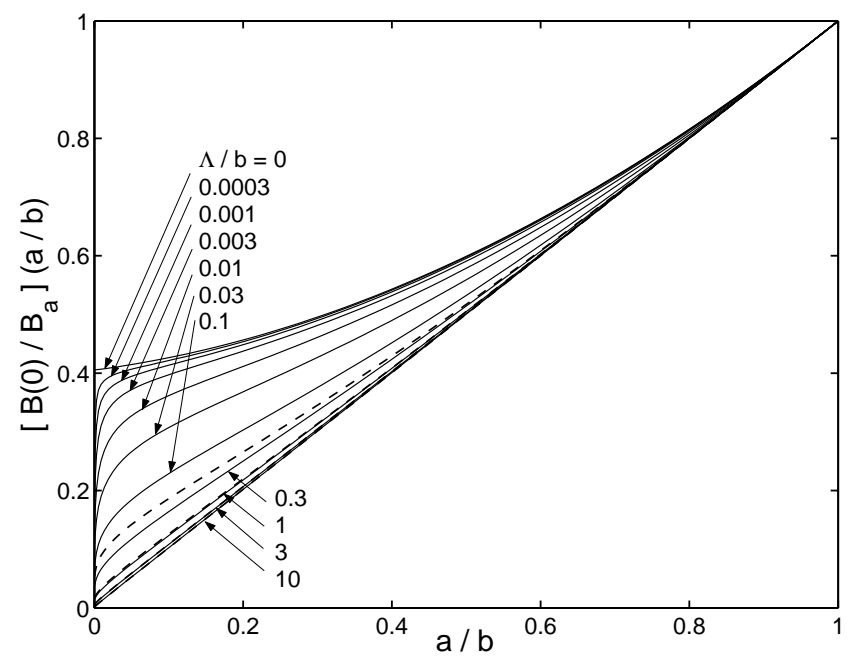

FIG. 16: Magnetic induction $B(0)$ in the center of a thin flat ring with $I=0$ (flux focusing) plotted as $\left[B(0) / B_{a}\right](a / b)$ versus $a / b$ for $\Lambda / b=0,0.0003,0.001,0.003,0.01,0.03,0.1$, $0.3,1,3$, and 10 . The dashed curves show the large- $\Lambda$ approximation, Eq. (48), for $\Lambda / b=0.3,1,3,10$.

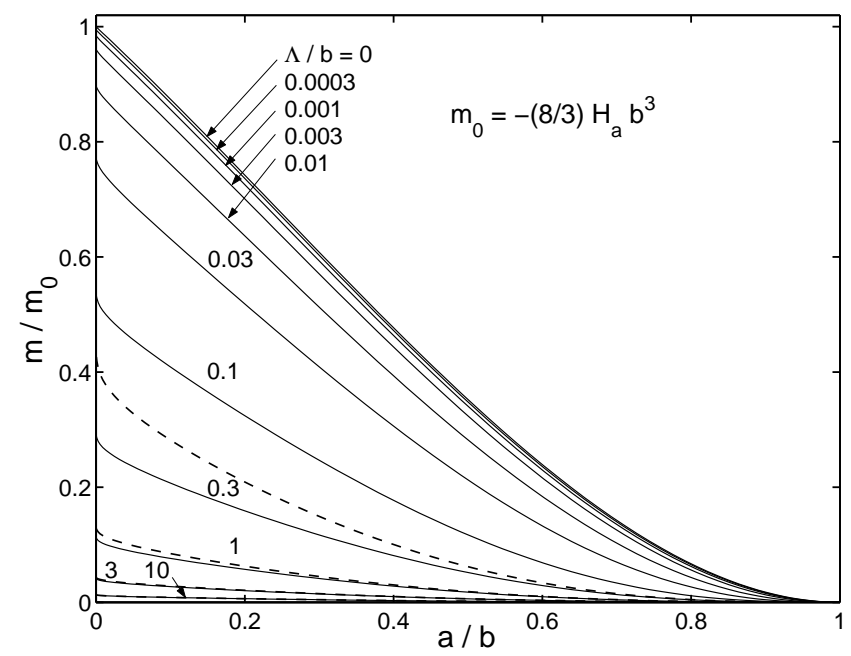

FIG. 17: Magnetic moment $m$ of a thin flat ring when $I=0$ (flux focusing) in units of $m_{0}=-(8 / 3) H_{a} b^{3}$ for $\Lambda / b=0$, $0.0003,0.001,0.003,0.01,0.03,0.1,0.3,1,3$, and 10 . The dashed curves show the large- $\Lambda$ approximation, Eq. (49), for $\Lambda / b=0.3,1,3,10$.

our approximation of a circularly symmetric ring the fluxoid $\Phi_{f}$ is given by $\Phi_{f}=2 \pi r\left[(r / 2) B_{a}+A_{J}(r)+\mu_{0} \Lambda J(r)\right]$ for any $r$ in the superconductor, $a \leq r \leq b$, and it equals the parameter $\Phi_{f}$ entering Eq. (2). In a superconductor without a slit, $\Phi_{f}=N \phi_{0}$ is quantized, with integer $N$. When the ring has a radial slit, it may be used to construct a dc SQUID by connecting the two banks of the slit to a superconducting current lead via two identical small Josephson junctions, each with maximum supercurrent $I_{0} \stackrel{12.13}{2}$ The critical current of the resulting dc 
SQUID is

$$
I_{c}=2 I_{0}\left|\cos \left(\pi B_{a} A_{\mathrm{eff}} / \phi_{0}\right)\right|,
$$

where the effective area is $A_{\text {eff }}=\Phi_{f} / B_{a}$. Note that the effective area can be calculated as $A_{\text {eff }}=\Phi(a) / B_{a}$ (as in Ref. 1) only in the limit $\Lambda=0$, when the magnetic flux $\Phi(a)$ in the hole of the ring is exactly equal to the fluxoid $\Phi_{f}$. The term "flux focusing" is appropriate because $A_{\text {eff }}$ is always larger than the actual area of the hole $\left(\pi a^{2}\right)$, regardless of the value of $\Lambda$; moreover, $A_{\text {eff }}$ is always in the range $\pi a^{2}<A_{\text {eff }}<\pi b^{2}$.

When $\Lambda=0$, one has the limits $1 \frac{1}{\text { eff }} /\left(\pi a^{2}\right)=$ $\left(8 / \pi^{2}\right)(b / a)$ for $a \ll b$ and $A_{\text {eff }} /\left(\pi a^{2}\right)=1+(1-a / b)$ for $a \rightarrow b$. A good fit valid for all $a$ in the range $0<a<b$ is (see Fig. 14)

$$
A_{\mathrm{eff}} /\left(\pi a^{2}\right)=1 /\left\{\tilde{a}\left[1+\left(\pi^{2} / 8-1\right)(1-\tilde{a})^{2}\right]\right\}
$$

with $\tilde{a}=a / b$. Values of $A_{\text {eff }}$ obtained from this expression deviate by less than $0.5 \%$ from the $\Lambda=0$ calculations of Ref. 1, 22 In the limit of large $\Lambda \gg b$ (which may be applicable to mesoscopic rings 9.23 .24 ) one has (see Eq. (46) below)

$$
A_{\text {eff }} /\left(\pi a^{2}\right)=\left(b^{2}-a^{2}\right) /\left[2 a^{2} \ln (b / a)\right],
$$

which is already closely approached for $\Lambda / b \geq 1$ (see Fig. 14).

The condition that $I=I_{1}+I_{2}=0$ (or $I_{2}=-I_{1}$ ) yields $A_{\text {eff }}=a b \beta_{I} / \alpha_{I}$, where $\beta_{I}=-\mu_{0} I_{2} / b B_{a}$, shown in Fig. 12, was computed in Sec. IV and $\alpha_{I}=\mu_{0} a I_{1} / \Phi_{f}$, shown in Fig. 6, was computed in Sec. III. Figure 13 shows the profiles of $J(r), B(r)$, and $A(r)$ for the case of flux focusing in a ring with $a / b=0.4$ for $\Lambda / b=0,0.03$, and 0.1. Note that the sheet current $J(r)$ changes sign inside the superconductor and has zero integral, $I=0$. As in Figs. 1 and 7, when $\Lambda=0, J(r)$ has inverse squareroot infinities at $r=a$ and $r=b$, which were treated in Ref. 1, but for $\Lambda>0, J(a)$ and $J(b)$ have finite values.

In Fig. 14 the reciprocal of the effective area $A_{\text {eff }}$ is plotted in the form $\pi a b / A_{\text {eff }}$, such that the data for all values of the penetration depth $0 \leq \Lambda / b \leq \infty$ can be presented in one plot. The curve for $\Lambda=0$ is well fitted by a parabola, Eq. (29), ranging from $\pi^{2} / 8$ at $a / b \rightarrow 0$ to 1 at $a / b \rightarrow 1$. The limiting curve for $\Lambda / b \gg 1$, Eq. (30), is practically reached already when $\Lambda / b$ exceeds unity. The main message from this plot is that $A_{\text {eff }}$ increases with increasing $\Lambda$ for any given $a / b$, and that for $\Lambda \neq 0$, $A_{\text {eff }} / \pi a b$ diverges when $a / b \rightarrow 0$, while for $\Lambda=0$ it tends to the finite value $8 / \pi^{2}$. For $\Lambda / b<0.03, A_{\text {eff }} / \pi a b$ has a minimum (since $\pi a b / A_{\text {eff }}$ has a maximum) as a function of $a / b$.

Figures 15, 16, and 17 show the magnetic flux $\Phi(a)$ in the hole of the ring plotted as $\Phi(a) / \pi a b B_{a}$, the magnetic field $B(0)$ in the center of the ring plotted as $\left[B(0) / B_{a}\right](a / b)$, and the magnetic moment $m / m_{0}$ of the ring for the flux-focusing case $I=0$. All three quantities decrease with increasing $\Lambda / b$.

\section{RING ENERGIES}

The energy of a superconducting ring with $\Lambda \gg b$ was calculated in Ref. 24 as a function of the fluxoid number $N$ and the applied magnetic induction $B_{a}$. Using the results of the previous sections, we are able to calculate the energy of a thin ring $(d<\lambda)$ with inner and outer radii $a$ and $b$ for any value of $\Lambda$. The authors of Ref. 24 also calculated, assuming $\Lambda \gg b$, the energy barriers between states $N$ and $N \pm 1$ associated with the energy cost of moving a vortex or antivortex between the inner and outer radii. However, to extend such calculations to the case of arbitrary $\Lambda$ is beyond the scope of our paper.

We begin by calculating the total electromagnetic energy of the ring-coil system, where the coil produces a perpendicular magnetic induction $B_{a}$ at the ring,

$$
E=\int d^{3} r \mathbf{B}^{2} / 2 \mu_{0}+\int d^{3} r \mu_{0} \lambda^{2} \mathbf{j}^{2} / 2 .
$$

The hole contains the fluxoid $\Phi_{f}=N \phi_{0}$, and $\mathbf{B}=$ $\mathbf{B}_{a}+\mathbf{B}_{J}$, as discussed in Sec. II. The first integral, which extends over all space, is the total magnetic-field energy, and the second integral, which extends only over the volume of the ring, is the total kinetic energy of the supercurrents. Using the vector potential, the divergence theorem, and Eqs. (2) and (4), we obtain

$$
E=E_{a}+m B_{a} / 2+N \phi_{0} I / 2,
$$

where $E_{a}=\int d^{3} r \mathbf{B}_{a}^{2} / 2 \mu_{0}$ is the applied field's magnetic energy in the absence of the ring, $m=m_{1}+m_{2}$ is the total magnetic moment of the ring, and $I=I_{1}+I_{2}$ is the total current around the ring. When the coil currents are controlled so as to maintain the magnetic induction $B_{a}$ applied to the ring, the relevant energy is the Gibbs free energy, which we define as $G_{N}\left(B_{a}\right)=E_{a}+g_{N}\left(B_{a}\right)=$ $E-W$, where $W=m B_{a}$ is the work done by the power supply in bringing up the magnetic moment to its final value $m$. Thus

$$
g_{N}\left(B_{a}\right)=-m B_{a} / 2+N \phi_{0} I / 2 .
$$

Eliminating $m$ and $I$ in favor of the quantities we have defined and calculated in Secs. II-V $\left(m_{1}=b N \phi_{0} \alpha_{m} / \mu_{0}\right.$, $m_{2}=-(8 / 3) b^{3} H_{a} \beta_{m}, I_{1}=N \phi_{0} \alpha_{I} / \mu_{0} a, I_{2}=-b H_{a} \beta_{I}$, $L=\mu_{0} a / \alpha_{I}$, and $\left.\beta_{I}=\alpha_{m}\right)$, we may express $g_{N}\left(B_{a}\right)$ in a form equivalent to that given in Ref. 24 for the case in which there are no vortices in the annular region $a<r<$ $b$ :

$$
g_{N}\left(B_{a}\right)=\epsilon_{0}\left[(h-N)^{2}+\gamma h^{2}\right] .
$$

The characteristic energy is $\epsilon_{0}=\phi_{0}^{2} / 2 L=\phi_{0}^{2} \alpha_{I} / 2 \mu_{0} a$. As can be seen most clearly from Fig. 2 , for given values of $a$ and $b, \epsilon_{0}$ decreases monotonically as $\Lambda$ increases from zero to $\infty$ (see Sec. VII); accordingly, $\epsilon_{0}$ is a monotonically decreasing function of temperature.

The reduced field in Eq. (34) is $h=B_{a} / B_{0}$, where the scaling field is $B_{0}=\phi_{0} / A_{\text {eff }}$, with $A_{\text {eff }}=a b \beta_{I} / \alpha_{I}$. Since 


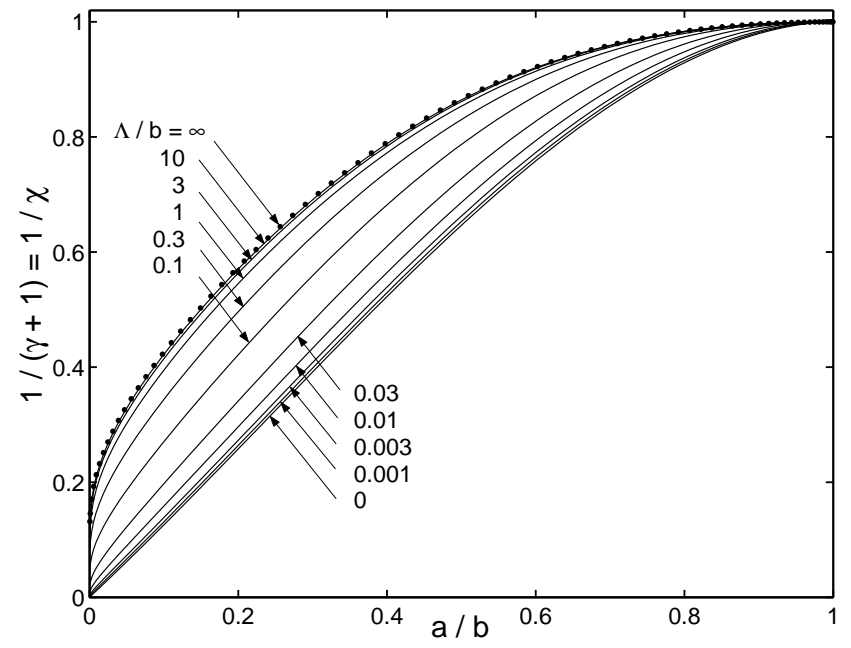

FIG. 18: Coefficient $\gamma$ of $h^{2}=\left(B_{a} / B_{0}\right)^{2}$ in the $N$ independent part of the energy of a superconducting ring in an applied field [see Eq. (34)], plotted as $1 /(\gamma+1)=1 / \chi$ to show the entire range of $0 \leq a / b \leq 1$. The dots mark the limit $\Lambda \rightarrow \infty$, Eq. (51).

$B_{0}$ is proportional to the reciprocal of $A_{\text {eff }}$, shown in Fig. 14 , we see that $B_{0}$ decreases from its largest value when $\Lambda=0$ to its smallest value when $\Lambda=\infty$. While $B_{0}$ is a monotonically decreasing function of temperature, the range of values spanned by $B_{0}$ is large only for small $a / b$; in a narrow ring for which $a \rightarrow b$, we find $B_{0} \rightarrow \phi_{0} / \pi b^{2}$, independent of $\Lambda$.

The constant $\gamma$ in the second term of Eq. (34), obtained as $\gamma=\chi-1=8 b \beta_{m} \alpha_{I} / 3 a \alpha_{m}^{2}-1$, is shown in Fig. 18. This $N$-independent quadratic term is relatively unimportant, however, because it is only the first term on the right-hand side of Eq. (34) that determines which quantum state $N$ has the lowest energy. Figure 19 exhibits a plot of $(h-N)^{2}$ vs $h=B_{a} / B_{0}$ for several values of $N$. From this plot we can see clearly that the state $N$ has the lowest energy for values of $h$ in the range $N-1 / 2<h<N+1 / 2$. When $h=N-1 / 2$, the states $N$ and $N-1$ have the same energy; similarly, when $h=N+1 / 2$, the states $N$ and $N+1$ have the same energy.

The temperature dependence of $B_{0}=\phi_{0} / A_{\text {eff }}$ leads to the possibility that the energetically favored value of $N$ may be higher close to $T_{c}$, where $\Lambda$ diverges, than at lower temperatures, where $\Lambda$ is much smaller. This effect, which may have important consequences for experiments using small superconducting rings, is greatest when $a / b$ is small. For example, suppose that $a=0.1 b$. At high temperatures for which $\Lambda / b>1$, we see from Fig. 14 that $A_{\mathrm{eff}} \approx 0.2 \pi b^{2}$, such that $B_{0} \approx 5 \phi_{0} / \pi b^{2}$. In an applied field of $B_{a} \approx 3 \phi_{0} / \pi b^{2}, h \approx 0.6$, and the energetically favored state is $N=1$. If at low temperatures $\Lambda / b<0.01$, then $A_{\text {eff }} \approx 0.1 \pi b^{2}$, about half that at high temperatures. Since the corresponding value of $B_{0}$ is then about twice that at high temperatures, $h \approx 0.3$, and the quantum number for the state with the lowest

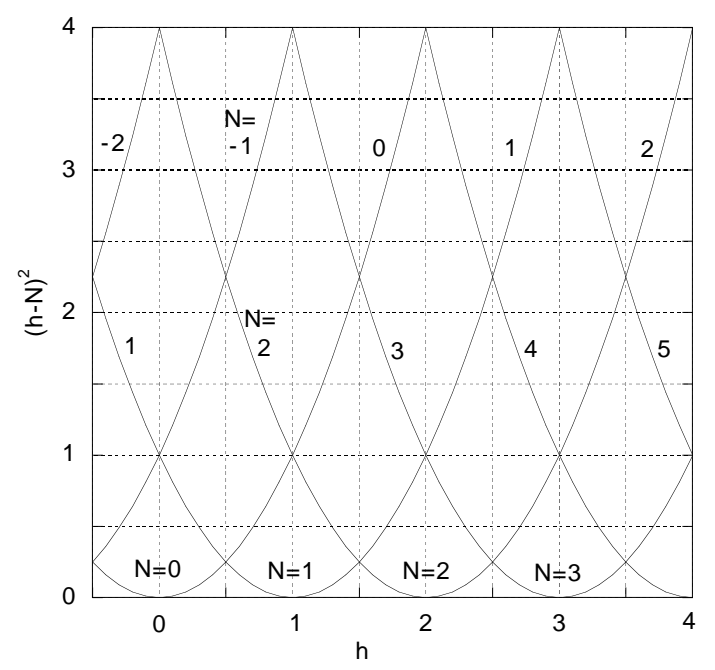

FIG. 19: $N$-dependent part of the energy of a superconducting ring in an applied field [see Eq. (34)], plotted as $(h-N)^{2}$ vs the reduced field $h=B_{a} / B_{0}$.

energy becomes $N=0$.

We emphasize that Eq. (34) gives the energy of a ring with fluxoid number $N$ in the hole when there are $n o$ vortices in the annular region $a<r<b$. It also states that the energetically favored value of $N$ is approximately equal to $B_{a} / B_{0}$ and hence increases as $B_{0}$ increases. However, since the intervortex spacing for an infinite thin film in an applied magnetic induction $B_{a}$ is of the order of $\left(\phi_{0} / B_{a}\right)^{1 / 2}$, we expect that it will first become energetically favorable for a vortex to sit in the middle of the annular region, rather than to enter the hole and increase the value of $N$, when $B_{a}=B_{c 1} \approx \phi_{0} / w^{2}$, where $w=b-a$. As pointed out in Ref. 24, for narrow rings $[w \ll(b-a)]$ when $\Lambda / b \gg 1$, $B_{c 1} \approx\left(2 \phi_{0} / \pi w^{2}\right) \ln (2 w / \pi \xi)$, where $\xi$ is the coherence length or vortex-core radius.

\section{LIMIT OF LARGE PENETRATION DEPTH}

In the limit $\Lambda / b \gg 1$, which can be realized in small mesoscopic rings, ${ }_{9,23,24}$ analytic expressions for many of the quantities of interest can be obtained from perturbation theory. To lowest order in the small parameter $b / \Lambda$, we may neglect the current-generated contribution $A_{J}$ to the vector potential $A$ in the London equation. From Eqs. (1) and (2) we thus obtain the sheet-current density $J:$

$$
J(r)=\frac{1}{\mu_{0} \Lambda}\left(\frac{\Phi_{f}}{2 \pi r}-\frac{1}{2} r B_{a}\right)
$$

From Eqs. (4) and the Biot-Savart law we then obtain the magnetic moment $m$, total current $I$, and magnetic induction at the center of the hole $b(0)$ generated by this 
current:

$$
\begin{gathered}
m=\frac{\pi}{\mu_{0} \Lambda}\left[\frac{\Phi_{f}}{4 \pi}\left(b^{2}-a^{2}\right)-\frac{B_{a}}{8}\left(b^{4}-a^{4}\right)\right], \\
I=\frac{1}{\mu_{0} \Lambda}\left[\frac{\Phi_{f}}{2 \pi} \ln \left(\frac{b}{a}\right)-\frac{B_{a}}{4}\left(b^{2}-a^{2}\right)\right], \\
b(0)=\frac{1}{2 \Lambda}\left[\frac{\Phi_{f}}{2 \pi} \frac{(b-a)}{a b}-\frac{B_{a}}{2}(b-a)\right],
\end{gathered}
$$

For the case $B_{a}=0$ and $\Phi_{f}>0$, the self-inductance $L$ is obtained from Eqs. (24) and (37). Expressing $L$ in terms of the same ratio as shown in Fig. 2, we obtain

$$
\frac{L}{\mu_{0} a}=\left(\frac{\Lambda}{b}\right) \frac{2 \pi}{(a / b) \ln (b / a)} .
$$

The inverse self-inductance, expressed as the dimensionless ratio shown in Fig. 6, is

$$
\alpha_{I}=\frac{\mu_{0} I_{1} a}{\Phi_{f}}=\frac{\mu_{0} a}{L}=\frac{1}{2 \pi}\left(\frac{b}{\Lambda}\right)\left(\frac{a}{b}\right) \ln \left(\frac{b}{a}\right) .
$$

Expressing the magnetic field $B_{1}(0)$ at the center of the ring in terms of the same ratio as plotted in Fig. 4, we obtain

$$
\frac{2 \pi a^{2} B_{1}(0)}{\Phi_{f}}=\frac{1}{2}\left(\frac{b}{\Lambda}\right)\left(\frac{a}{b}\right)\left(1-\frac{a}{b}\right) .
$$

The magnetic moment $m_{1}$ expressed as the same dimensionless ratio plotted in Fig. 5, is

$$
\alpha_{m}=\frac{\mu_{0} m_{1}}{b \Phi_{f}}=\frac{1}{4}\left(\frac{b}{\Lambda}\right)\left[1-\left(\frac{a}{b}\right)^{2}\right] .
$$

For the zero-fluxoid case, we obtain the magnetic induction $B_{2}(0)$ at the center of the ring by setting $\Phi_{f}=$ 0 and adding $B_{a}$ to Eq. (38). Expressing the result in terms of the same ratio as plotted in Fig. 9, we obtain

$$
\frac{B_{2}(0)}{B_{a}}=1-\frac{1}{4}\left(\frac{b}{\Lambda}\right)\left(1-\frac{a}{b}\right) .
$$

The corresponding magnetic moment is obtained from Eq. (36) with $\Phi_{f}=0$. Expressing the result in terms of the same dimensionless ratio as plotted in Fig. 11, we obtain

$$
\beta_{m}=\frac{m_{2}}{m_{0}}=\frac{3 \pi}{64}\left(\frac{b}{\Lambda}\right)\left[1-\left(\frac{a}{b}\right)^{4}\right] .
$$

The total current in the ring is obtained from Eq. (37), again with $\Phi_{f}=0$. Expressing the result in terms of the same dimensionless ratio as plotted in Fig. 12, we obtain

$$
\beta_{I}=-\frac{I_{2}}{b H_{a}}=\frac{1}{4}\left(\frac{b}{\Lambda}\right)\left[1-\left(\frac{a}{b}\right)^{2}\right] .
$$

This result is the same as that in Eq. (42), as expected from the identity $\alpha_{m}=\beta_{I}$, proved earlier.
For the case of flux focusing, we obtain the effective area $A_{\text {eff }}=\Phi_{f} / B_{a}$ by setting $I=0$ in Eq. (37). The result is

$$
A_{\mathrm{eff}}=a b \frac{\beta_{I}}{\alpha_{I}}=\frac{\pi\left(b^{2}-a^{2}\right)}{2 \ln (b / a)} .
$$

Expressing $A_{\text {eff }}$ in terms of the same ratio as plotted in Fig. 14, we obtain

$$
\frac{\pi a b}{A_{\mathrm{eff}}}=\pi \frac{\alpha_{I}}{\beta_{I}}=\frac{2(a / b) \ln (b / a)}{1-(a / b)^{2}} .
$$

The corresponding magnetic induction at the center of the ring $B(0)$ is obtained by adding $b(0)$ [Eq. (38)] to $B_{a}$ but using Eq. (37) and $I=0$ to eliminate $\Phi_{f}$. Expressing the result in terms of the same ratio as plotted in Fig. 16, we obtain

$$
\frac{B(0)}{B_{a}} \frac{a}{b}=\frac{a}{b}+\frac{1}{4}\left(\frac{b}{\Lambda}\right)\left(1-\frac{a}{b}\right)\left[\frac{\left(1-a^{2} / b^{2}\right)}{2 \ln (b / a)}-\frac{a}{b}\right] .
$$

The corresponding magnetic moment $m$ is obtained from Eq. (36), using Eq. (37) and $I=0$ to eliminate $\Phi_{f}$. Expressing the result in terms of the same ratio as plotted in Fig. 17, we obtain

$$
\frac{m}{m_{0}}=\frac{3 \pi}{64}\left(\frac{b}{\Lambda}\right)\left[1-\left(\frac{a}{b}\right)^{2}\right]\left[1+\left(\frac{a}{b}\right)^{2}-\frac{\left(1-a^{2} / b^{2}\right)}{\ln (b / a)}\right] .
$$

Finally, we calculate the quantities of interest for ring energies, discussed in Sec. VI. When $\Lambda / b \gg 1$, the characteristic energy, $\epsilon_{0}=\phi_{0}^{2} / 2 L=\phi_{0}^{2} \alpha_{I} / 2 \mu_{0} a$, becomes [see Eq. (40)]

$$
\epsilon_{0}=\frac{\phi_{0}^{2} \ln (b / a)}{4 \pi \mu_{0} \Lambda},
$$

which agrees with that found in Ref. 24, allowing for the different system of units and definition of $\Lambda$ used there. For large $\Lambda$, the quantity $\gamma=\chi-1=8 b \beta_{m} \alpha_{I} / 3 a \alpha_{m}^{2}-1$ appearing in Eq. (34) becomes

$$
\gamma=\frac{1+(a / b)^{2}}{1-(a / b)^{2}} \ln (b / a)-1,
$$

in agreement with Ref. 24 .

Although the results presented in this section should apply only when $\Lambda / b \gg 1$, comparison of the above expressions with the numerical results show that they provide good approximations already when $\Lambda / b=1$ and yield excellent results when $\Lambda / b \geq 3$.

\section{SUMMARY}

In this paper we presented a straightforward matrixinversion method for the solution of the sheet current, vector potential, and magnetic field generated by a thinfilm $(d<\lambda / 2)$ superconducting ring (inner and outer 
radii $a$ and $b$ ) containing a trapped fluxoid $\Phi_{f}$ in a perpendicular applied magnetic induction $B_{a}$ for values of $\Lambda=\lambda^{2} / d$ ranging from zero to infinity. We used this method to calculate magnetic-field, current-density, and vector-potential profiles and numerous related physical quantities for three important cases: (i) $\Phi_{f}>0$ but $B_{a}=0$ (trapped fluxoid, index $n=1$ ), (ii) $B_{a}>0$ but $\Phi_{f}=0$ (zero-fluxoid state, index $n=2$ ), and (iii) $\Phi_{f}>0$ and $B_{a}>0$ but no net current around the ring (flux focusing). We also calculated the Gibbs free energy of the ring as a function of the quantum number $N$, where $N=\Phi_{f} / \phi_{0}$, and the applied magnetic induction
$B_{a}$ when no vortices are present in the annular region between $a$ and $b$.

\section{Acknowledgments}

This work was supported in part by the German Israeli Research Grant Agreement (GIF) No G-705-50.14/01 and in part by Iowa State University of Science and Technology under Contract No. W-7405-ENG-82 with the U.S. Department of Energy.
1 A. A. Babaei Brojeny and J. R. Clem, Phys. Rev. B. 68, 174514 (2003).

2 E. H. Brandt, Phys. Rev. B 50, 4034 (1994).

3 J. Gilchrist and E. H. Brandt, Phys. Rev. B 54, 3530 (1996).

4 E. H. Brandt, Phys. Rev. B 58, 6506 (1998).

5 E. H. Brandt, Phys. Rev. B 55, 14513 (1997).

${ }^{6}$ M. Pannetier, F. C. Klaasen, R. J. Wijngaarden, M. Welling, K. Heeck, J. M. Huijbregtse, B. Dam, and R. Griessen, Phys. Rev. B 64, 144505 (2001).

7 T. W. B. Kibble, J. Phys. A 9, 1387 (1976).

8 W. H. Zurek, Nature (London) 317, 505 (1985); Phys. Rep. 276, 177 (1996).

9 J. R. Kirtley, C. C. Tsuei, and E. Tafuri, Phys. Rev. Lett. 90, 257001 (2003).

10 R. Carmi and E. Polturak, Phys. Rev. B 60, 7595 (1999); A. Maniv, E. Polturak, and G. Koren, Phys. Rev. Lett. 91, 197001 (2003).

11 M. B. Ketchen, W. J. Gallagher, A. W. Kleinsasser, S. Murphy, and J. R. Clem, in SQUID '85, Superconducting Quantum Interference Devices and their Applications, edited by H. D. Hahlbohm and H. Lübbig (de Gruyter, Berlin, 1985), p. 865.
12 T. P. Orlando and K. A. Delin, Foundations of Applied Superconductivity (Addison-Wesley, Reading, 1991).

13 A. Barone, ed., Principles and Applications of Quantum Interference Devices (World Scientific, Singapore, 1992).

14 D. Koelle, R. Kleiner, F. Ludwig, E. Dantsker, and John Clarke, Rev. Mod. Phys. 71, 631 (1999).

15 F. London, Superfluids, Vol. 1 (Dover, New York, 1961).

16 E. H. Brandt, Phys. Rev. B 64, 024505 (2001).

17 The constancy of the London fluxoid $2 \pi r\left[A_{J}(r)+\mu_{0} \Lambda J(r)\right]$ is confirmed by our matrix-inversion method (written in MATLAB) with high precision, to 15 significant digits for all $a \leq r \leq b$.

18 J. Pearl, Appl. Phys. Lett. 5, 65 (1964).

19 J. R. Clem, Phys. Rev. B 43, 7837 (1991).

${ }^{20}$ G. Carneiro and E. H. Brandt, Phys. Rev. B 61, 6370 (2000)

21 J. R. Clem and A. Sanchez, Phys. Rev. B 50, 9355 (1994).

22 A. A. Babaei Brojeny, private communication.

23 J. R. Kirtley, C. C. Tsuei, V. G. Kogan, J. R. Clem, H. Raffy, and Z. Z. Li, Phys. Rev. B 68, 214505 (2003).

24 V. G. Kogan, J. R. Clem, and R. G. Mints, cond-mat/0310393 (16 Oct. 2003). 